\title{
Importancia de la ruta de parto en la adquisición del microbioma en la temprana infancia
}

\author{
Importance of the delivery route in the acquisition \\ of the microbiome in early childhood
}

\author{
Marlene Ortiz M. ${ }^{a}$, Paul R Harris ${ }^{\mathrm{a}}$
}

aDepartamento de Gastroenterología y Nutrición Pediátrica, Facultad de Medicina,

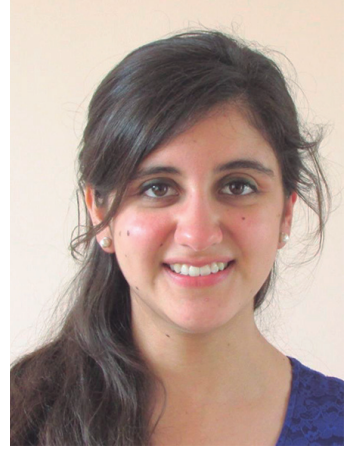

Pontificia Universidad Católica de Chile

En los últimos 15 años se ha descrito un aumento en la tasa de cesáreas a nivel mundial, alcanzando un $30 \%$ en EE.UU. y sobrepasando el $40 \%$ en Chile. Múltiples estudios demuestran este aumento sostenido, siendo el caso de Chile mayor al de otros países de la región y mayor a la mayoría de los países pertenecientes a la OCDE $\mathrm{O}^{1}$. La causa de este aumento pareciera ser multifactorial y muy complejo. Más allá de sus orígenes y de la interacción entre factores que participan en este resultado, surge la preocupación sobre el impacto que ello pudiera tener en el desarrollo de distintas enfermedades durante la niñez. De hecho, múltiples estudios de asociación demuestran un mayor riesgo de enfermedades alérgicas (asma, rinitis alérgica), inmunológicas (enfermedad de Crohn, enfermedad celíaca), metabólicas (diabetes mellitis tipo 1) y obesidad en niños nacidos vía cesárea, comparados con niños nacidos por ruta vaginal ${ }^{2}$. Los mecanismos biológicos que subyacen a estas asociacioness comienzan a despejarse con el estudio reciente de la adquisición temprana de la microbiota por el recién nacido.

La dinámica ecológica de las bacterias que conforman la microbiota humana y la totalidad de la información genética contenida en ellas (microbioma), han sido difíciles de estudiar principalmente por los desafíos técnicos asociados a la naturaleza de cada bacteria (la mayoría de muy difícil cultivo). Sin embargo, con la aparición de nuevas técnicas de secuenciación de última generación (NGS) que utilizan la filogenia de la subunidad $16 \mathrm{~S}$ rRNA, junto a plataformas bioinformáticas complejas, se ha logrado conocer la composición de la microbiota y su rol en salud y enfermedad. Fue así, que, en el año 2012, se completó The Human Microbiome Project, donde se describió por primera vez la diversidad de composición del microbioma humano en adultos sanos de Estados Unidos ${ }^{3}$.

Debieron pasar algunos años más para conocer las diferencias en la colonizacion de los recién nacidos dependiente de la ruta de parto. Así en su paso por el canal del parto, los recién nacidos adquieren distintas bacterias ácido-lácticas, como Lactobacillus, Prevotella y Sneathia spp. de la microbiota vaginal de la madre. Aunque solo algunos de estos colonizadores permanecerán en los lactantes, la exposición inicial es un punto clave para el desarrollo de la microbiota adulta. Por otro lado, los grupos de bacterias colonizadoras pioneras de recién nacidos por cesárea corresponden al tipo Staphylococcus, Corynebacterium y Propionibacterium spp, que provienen de la microbiota de la piel de la madre ${ }^{4}$.

Para entender el microbioma en un recién nacido, este debe relacionarse con la composición del micro-

Correspondencia:

Paul R. Harris

pharris@med.puc.cl 
bioma de su madre, ya que existe una profunda interconexión. Se pensaba al feto como esencialmente estéril hasta que ocurre la ruptura de las membranas ovulares o saco amniótico, pero se ha visto la presencia, a través del estudio de microbiota en meconio del recién nacido, de Escherichia y Klebsiella, conocidos anaerobios facultativos tempranos del tracto gastrointestinal, no vistos inicialmente en otras partes del cuerpo del neonato, pero sí descritas en la placenta y líquido amnniótico a través de secuanciación del 16S rRNA. Posterior al nacimiento, la microbiota materna del tracto vaginal y tracto urinario (o piel en caso de cesárea), cavidad oral e intestino, contribuyen a la siembra inicial de la microbiota de neonato, como se describió previamente. Ciertamente existen múltiples otros factores que condicionan la composición y diversidad de la microbiota de un recién nacido como son factores maternos (IMC, infecciones previas, uso de antibióticos, dieta materna, entre otros) y factores perinatales (principalmente disponibilidad de lactancia materna y uso precoz de antibióticos). Sin embargo, los recién nacidos son principalmente inoculados al nacer a través del paso por el canal del parto, por transmisión vertical ${ }^{5}$.

Posteriormente, en la vida del lactante debieran ocurrir variadas exposiciones que modificarían teóricamente la microbiota inicial, conocido como transmisión horizontal de microbiota, siendo la microbiota de un niño de 2 años indistinguible de la microbiota de un adulto. Sin embargo, se postula que durante las últimas décadas, los cambios de estilos de vida en el mundo occidental y cambios en el ambiente (disminución del tamaño de las familias, pérdida de lactancia materna, excesiva sanitización del ambiente, uso de antibióticos, entre otros) estarían contribuyendo a alterar esta adquisición horizontal de microbiota, que no sería capaz de reponer las pérdidas en la adquisicion vertical de microbiota en las sucesivas generaciones.
Finalmente una alteración en la trasmisión vertical no equilibrada por una teórica compensación con la adquisión horizonatal de microbiota contibuiría a generar una microbiota anómala o disbiótica. La interacción de esta microbiota disbiótica con el desarrollo temprano del sistema inmune en el lactante y sus mecanismos de regulación están en activo estudio con la conformación de múltiples cohortes poblacionales que consideran el estudio de factores ambientales, epigenéticos, inmunoregulatorios y de microbioma desde el período fetal, incorporándose al concepto de la programación prenatal de las enfermades y a la teoría de los primeros 1.000 días, como el periodo critico en la determinación o predisposición al desarrollo de una serie de enfermedades de tipo inflamatorio, autoinmune y metabólicos, tales como asma, diabetes, obesidad y enfermedades gastrointestinales crónicas, alarmantemente cada vez más prevalentes en occidente ${ }^{6}$.

Por lo anteriormente expuesto, es que cobra relevancia el estudio colaborativo internacional para evaluar los cambios de la microbiotas desde el período de recién nacido, qué factores influyen en ella, y como estos cambios se correlacionan con el diagnóstico inicial de distintas enfermedades de la infancia ${ }^{7}$. De este modo, se podría intervenir de manera temprana en medidas que contribuyesen a prevenir la aparición de éstas.

\section{Financiamiento}

CONICYT-PIA ANILLO (ACT172097).

\section{Conflicto de intereses}

Los autores declaran no tener conflicto de intereses.

\section{Referencias}

1. OMS, Organización Mundial de la Salud. Declaración de la OMS sobre tasa de cesárea. Suiza: OMS, 2015.

2. Biedermann L, Rogler G. The intestinal microbiota: its role in health and disease. Eur J Pediatr. 2015;174:151-67.

3. Cho I, Blaser, M. The human microbiome: at the interface of health and disease. Nat Rev Genet. 2012;13:260-70.
4. Domínguez-Bello MG, Costello EK, Contreras M, et al. Delivery mode shapes the acquisition and structure of the initial microbiota across multiple body habitats in newborns. Proc Natl Acad Sci USA. 2010;107:11971-5.

5. Chu DM, Ma J, Prince AL, Antony KM, Seferovic MD, Aagaard KM. Maturation of the infant microbiome community structure and function across multiple body sites and in relation to mode of delivery. Nature Medicine. 2017; doi:10.1038/nm.4272.

6. DuPont AW, DuPont HL. The intestinal microbiota and chronic disorders of the gut. Nature reviews. Gastroenterology \& hepatology. 2011;8:523-31.

7. Hill CJ, Lynch DB, Murphy K, et al. Evolution of gut microbiota composition from birth to 24 weeks in the INFANTMET Cohort. Microbiome. 2017; 5:4. doi:10.1186/s40168-016-0213-y. 